Relato de Experiência

\title{
Efeito do treinamento resistido em paciente com fibromialgia: Estudo de caso
}

\author{
Vanessa Zadorosnei Rebutini ${ }^{1}$ \\ Marilia Tumelero Giaretta ${ }^{2}$ \\ Jeanne Rissato da Silva ${ }^{2}$ \\ Andrea Karla da Silva Mayork ${ }^{2}$ \\ César Cavinato Cal Abad ${ }^{3}$ \\ ${ }^{1}$ Centro de Estudos de Comportamento Motor, Universidade Federal \\ do Paraná, Curitiba, PR, Brasil \\ ${ }^{2}$ Universidade Gama Filho; Curitiba, PR, Brasil \\ ${ }^{3}$ UNIBAN-Anhaguera, São Paulo, SP, Brasil
}

\begin{abstract}
Resumo: A fibromialgia (FM) é uma doença idiopática de ordem reumatológica e incurável que causa dores crônicas e possui tratamento complexo e multifatorial. O exercício físico pode fazer parte do tratamento por atenuar parte dos sintomas da FM, porém a literatura ainda carece de informações sobre tipo, intensidade, duração e frequência ótimos contra os sintomas da FM. O objetivo do presente estudo foi verificar o efeito de 12 semanas de treinamento resistido em uma paciente com FM que não fazia tratamento farmacológico. Os resultados indicaram que 12 semanas de treinamento resistido foi capaz de reduzir as dores, melhorar a capacidade funcional, o bem-estar geral e a qualidade de vida da paciente. $O$ treinamento resistido pareceu atenuar os efeitos negativos da FM e parece ser uma ferramenta promissora para pacientes com FM que gostem desse tipo de atividade.
\end{abstract}

Palavras-chave: Treinamento Resistido. Fibromialgia. Qualidade de vida..

\section{Effect of resistance training in patients with fibromyalgia: A case report}

\begin{abstract}
Fibromyalgia (FM) is an idiopathic rheumatological order and incurable desease that causes chronic pain and has complex and multifactorial treatment. Exercise may be a part of treatment by relieving some of the symptoms of FM, but the literature still lacks optimal information about type, intensity, duration and frequency against the symptoms of FM. The aim of this study was to verify the effect of 12 weeks of resistance training in a patient with FM that had no pharmacological treatment. The results indicated that 12 weeks of resistance training was able to reduce pain, improve functional capacity, general well-being and quality of life of the patient. Resistance training appeared to attenuate the negative effects of FM and seems to be a promising tool for patients with FM who enjoy this kind of activity.
\end{abstract}

Keywords: Resistance Training. Fibromyalgia. Quality of Life.

\section{Introdução}

A fibromialgia (FM) é uma doença idiopática de ordem reumatológica e incurável que causa dores crônicas dispersas em todo o corpo. Além da dor difusa o paciente também pode apresentar rigidez dos músculos e articulações (ANG et al., 2011), redução da força e da resistência musculares (KINGSLEY et al., 2005; PANTON et al., 2006), intolerância ortostática (RAJ et al., 2000) intolerância ao frio (QIAO et al., 1991) fadiga (PANTON et al., 2006; WIERWILLE, 2011), hiperalgesia, alodinia, distúrbios do sono, ansiedade, depressão, dor de cabeça, disfunção intestinal, transtornos mentais e físicos, queda na qualidade de vida (HAUSER et al., 2010b; BELLATO et al., 2012), somação temporal de estímulos nociceptivos (STAUD; CANNON, 2003; GOLDENBERG et al., 2004) prejuízos na atenção e cognição e problemas de humor ( $\underline{\mathrm{ANG}}$ et al., 2011).

Com causa desconhecida, de caráter multifatorial e com difícil tratamento, vários aspectos podem estar associados com o desencadeamento da FM. Disfunção do sistema nervoso autônomo (BELLATO et al., 2012) com alterações nos padrões basais de eixos neuroendócrinos em resposta ao estresse (MARTINEZ-LAVIN, 2002), polimorfismos do gene da serotonina e da enzima catecol-ometiltransferase (que inativa as catecolaminas) (OFFENBAECHER et al., 1999; GURSOY et al., 2003) e fatores psicossociais juntamente com aspectos familiares e outras co-morbidades parecem ser os fatores que mais contribuem para a manifestação clínica da FM (WIERWILLE, 2011). 
Para diagnóstico da $\mathrm{FM}$, o critério mais utilizado e aceito na pesquisa clínica é o recomendado pelo Colégio Americano de Reumatologia que preconiza o histórico de dor bilateral em membros superiores, inferiores e na espinha dorsal por pelo menos três meses e também o excesso de sensibilidade à dor na aplicação de pressão em onze dos dezoito pontos músculo-tendíneos específicos (WOLFE et. al.,1990).

Acredita-se que nos Estados Unidos a FM seja o segundo maior distúrbio observado por reumatologistas perdendo apenas para osteoartrite (CROFFORD; CLAUW, 2002; WIERWILLE, 2011). Estudos de base populacional estimam que a prevalência da FM varia de 0,5 a $5,8 \%$, afetando $3,4 \%$ das mulheres e $0,5 \%$ dos homens (HAUSER et al., 2010b; $\underline{\text { ANG }}$ et al., 2011). Segundo Lawrence et al., (2008), a FM afeta aproximadamente oito vezes mais mulheres do que homens, e num estudo canadense de grande escala constatou-se FM auto-relatada em $1,83 \%$ das mulheres contra $0,33 \%$ dos homens (MCNALLY et al., 2006). Esses dados assemelham-se aos de Gilliland 2005 e Wolfe et al., 1995 que destacam prevalência de $\mathrm{FM}$ quase nove vezes maior em mulheres do que em homens e que a FM aumenta sua prevalência com a idade.

Existem variadas opções de tratamento farmacológico (amitriptilina, tramadol, fluoxetina, venlafaxina, milnacipran: duloxetina regabalin) e não-farmacológicos (exercício físico, terapia cognitivo-comportamental, programa educacional e terapia múltipla) (SANUDO et al., 2010) disponíveis para os portadores de FM, porém a gestão ótima da doença ainda é desconhecida. Alguns pesquisadores, inclusive, acreditam que não exista um único tratamento que seja eficaz para a FM (EHRLICH, 2003).

Nos Estados Unidos, menos de $20 \%$ dos médicos cuidam dos pacientes com FM (CROFFORD; CLAUW, 2002). Entre as justificativas para o baixo índice de tratamento está a falta de diretrizes específicas no manejo da doença devido às controvérsias encontradas na literatura. Em uma revisão sistemática baseada em ensaios clínicos controlados, por exemplo, concluiu-se que a terapia múltipla permanente é eficaz para diminuição da dor e melhora da aptidão física (BURCKHARDT, 2006) enquanto outra revisão sistemática baseada em estudo randomizado com ensaio controlado concluiu que os benefícios da terapia múltipla são limitados já que os benefícios desaparecem em longo prazo (KOULIL, 2007). Além dessa controvérsia, a orientação da Sociedade Americana de Dor atribui a terapia múltipla com nível de recomendação alta (BURCKHARDT et al., 2005), enquanto que o Comitê Europeu Multidisciplinar a recomenda somente baseada na opinião de especialistas (CARVILLE et al., 2008).

O exercício físico pode ser utilizado como uma estratégia não-medicamentosa para o tratamento da FM (ELLINGSON et al., 2012) e os mais utilizados são os aeróbicos (JONES; LIPTAN, 2009) que parecem reduzir dor, fadiga, depressão, melhorar a saúde, a qualidade de vida e a aptidão física dos pacientes com FM (HAUSER et. al., 2010a). Mais recentemente Hurley et al., 2011 demonstraram que o treino de força pode ser efetivo para reverter efeitos adversos da FM. Porém, como seu tratamento é multifatorial e diversificado, muitos estudos apresentam problemas metodológicos que comprometem conclusões mais apuradas

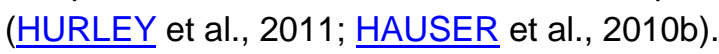

Parte do problema parece ocorrer em função das amostras mais numerosas serem heterogêneas e trazerem resultados inconclusivos devido às várias formas de tratamento utilizados por cada indivíduo (HAUSER et al., 2010b). Além disso, em amostras homogêneas os resultados acabam não apresentando poder estatístico e impossibilitam extrapolações populacionais. Em

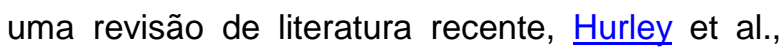
(2011) corroboram com afirmativa de que a maioria dos estudos que utilizaram o treinamento resistido como tratamento da $\mathrm{FM}$ era de baixa qualidade. Por outro lado, autores de pelo menos 3 estudos discordam e afirmam que programas de treinamento resistido, executados corretamente, são seguros e podem ser recomendados para pacientes com FM (JONES et al., 2002; HAKKINEN et al., 2001; VALKEINEN et al., 2005).

Devido à falta de informações sobre a modalidade ideal de treinamento e todos os problemas metodológicos já destacados, estudos que tragam informações adicionais sobre o treinamento físico em pacientes com FM devem ser realizados.

O objetivo do presente trabalho foi relatar o comportamento da força e da percepção subjetiva 
de dor de uma paciente com FM submetida a um programa de treinamento resistido tradicional por 12 semanas. Acreditamos que esse tipo de estudo, apesar de apresentar algumas limitações, pode trazer informações relevantes, especialmente do ponto vista prático quando os profissionais envolvidos com o tratamento de FM devem decidir quais estratégias utilizar.

\section{Métodos}

A voluntária foi informada sobre os riscos do estudo e assinou o termo de consentimento livre e esclarecido. $O$ estudo foi aprovado pelo comitê de ética local (protocolo 1409) e todos os procedimentos seguiram a resolução 196/96 do Conselho Nacional de Saúde de estudos com seres humanos.

A voluntária possuía 52 anos, $64 \mathrm{Kg}$ de massa corpórea, $168 \mathrm{~cm}$ de estatura e IMC de 22,6. Na ausência dos sintomas da FM a paciente praticava esporadicamente atividades aeróbicas leves ou moderadas (caminhada, bicicleta ou esteira ergométrica; 50 a $60 \%$ da frequência cardíaca de reserva de 20 a $40 \mathrm{~min}$ ). Além disso, ela não realizava nenhum tipo de tratamento não medicamentoso contra FM. O diagnóstico da FM foi feito pelo método de concentração de pontos sensíveis havia 2 anos e a paciente relatava maior queixa de pontos sensíveis na região dorsal e na cintura escapular (especificamente trapézio e supra-espinal).

O treinamento constou de 12 semanas de exercícios resistidos subdivididas em três sessões semanais de 40 minutos em dias alternados. A sessão foi distribuída em: aquecimento em cicloergômetro (6 minutos; com intensidade entre
9 e 11 na Escala de Borg), treinamento resistido (40 segundos para a execução das repetições, com intervalo de 45 segundos entre as séries) e alongamento (de intensidade leve, com aproximadamente 15 segundos de execução em cada porção muscular). Foram prescritos 8 exercícios, distribuídos alternadamente por segmento, com séries planejadas de forma progressiva.

A primeira semana constou de adaptação com série única de 10 repetições; a segunda e a terceira semanas constaram de 2 séries de 10 repetições; da quarta à décima segunda semanas foram realizadas 3 séries de 10 repetições. A carga foi incrementada conforme a escala de esforço percebido pela voluntária, com indicações de grau 6 na Escala de Borg. Tal procedimento ocorreu conforme observações já feitas sobre o comportamento da doença em estudos prévios (BUSCH et al., 2008; RIBEIRO; MARINHO, 2005) (Tabela1). Todos os procedimentos seguiram as recomendações do American College of Sports Medicine ACSM e do Consenso Brasileiro de Fibriomialgia (HEYMANN et al., 2010).

A certificação do estado de saúde da voluntária foi verificada por anamnese e pelo Questionário de Prontidão para Atividade Física (PAR-Q).

As demais avaliações foram divididas em controle e acompanhamento e foram aplicados os instrumentos de FIQ (Fibromyalgia Impact Questionnaire) (MARQUES et. al., 2006) e de NAHAS et al., (2000) no início do programa, na quarta, oitava e na décima-segunda semana, Ambos os questionários foram aplicados na forma de entrevista e as respostas anotadas.

Tabela 1. Programa de treinamento resistido com descritivo da carga.

\begin{tabular}{|c|c|c|c|c|c|c|c|c|c|c|c|c|}
\hline \multirow{2}{*}{ Exercício } & \multicolumn{12}{|c|}{ Semanas } \\
\hline & 1 & 2 & 3 & 4 & 5 & 6 & 7 & 8 & 9 & 10 & 11 & 12 \\
\hline Leg Press $45^{\circ}(\mathrm{kg})$ & 40 & 60 & 65 & 65 & 65 & 65 & 65 & 70 & 75 & 75 & 75 & 75 \\
\hline Remada Aberta (kg) & 20 & 23 & 25 & 25 & 25 & 25 & 25 & 25 & 25 & 25 & 25 & 25 \\
\hline Mesa Flexora (kg) & 5 & 12 & 15 & 15 & 15 & 15 & 15 & 15 & 15 & 15 & 15 & 15 \\
\hline Supino Máquina (kg) & $5+5$ & $6+6$ & $6+6$ & $6+6$ & $6+6$ & $6+6$ & $6+6$ & $6+6$ & $7+7$ & $7+7$ & $7+7$ & $7+7$ \\
\hline Extensor (kg) & 20 & 20 & 25 & 25 & 25 & 25 & 25 & 25 & 25 & 25 & 25 & 25 \\
\hline Rosca Cross (kg) & 10 & 15 & 15 & 15 & 15 & 15 & 15 & 15 & 15 & 15 & 15 & 15 \\
\hline Panturrilha Leg (kg) & 40 & 55 & 55 & 55 & 55 & 55 & 55 & 55 & 60 & 60 & 60 & 60 \\
\hline Triceps Pulley (kg) & 15 & 15 & 15 & 15 & 15 & 15 & 15 & 15 & 15 & 15 & 15 & 15 \\
\hline
\end{tabular}


A partir desses dados observou-se a evolução da voluntária na atividade não somente em relação às condições físicas, mas também em relação às respostas da sobrecarga.

A Escala de Faces (escala numérica com seis níveis de percepção de dor) foi aplicada com o intuito de verificar o comportamento da dor em relação à prática do treinamento físico, e para tal foi aplicado pré e pós-sessão em todos os dias de treino.

\section{Resultados}

A voluntária descreveu suas condições iniciais de dor devido à inatividade física, porém afirmou ter melhorado a qualidade de sono. Ao final das doze semanas do programa de treinamento ela relatou que o sono havia melhorado muito e que as dores haviam diminuído tanto no estado de repouso quanto nas sessões de treino e nas tarefas da vida diária. Também houve relato de melhorias na execução de atividades com requisição de membros superiores e de tarefas gerais que necessitavam de ações repetitivas ou cíclicas, como: lavar louças, varrer a casa e arrumar prateleiras.

A tabela 2 apresenta o comportamento da escala de faces à qual descreve as respostas da percepção subjetiva de dor apontada imediatamente antes e uma hora após o término da sessão de treinamento resistido. É possível verificar que nas primeiras duas semanas houve apenas respostas de dor de nível intermediário a intenso. Nas duas semanas seguintes, houve variação dessa resposta entre a dor intermediária e moderada-leve e nas demais semanas a resposta predominante foi moderada-leve.

Tabela 2. Percepção de dor da voluntária segundo Escala de Faces, antes (a) e uma hora depois (d) da sessão de treino.

\begin{tabular}{|c|c|c|c|c|c|c|c|c|c|c|c|c|c|c|c|c|c|c|c|c|c|c|c|c|}
\hline & \multicolumn{24}{|c|}{ Semanas } \\
\hline & \multicolumn{2}{|c|}{1} & \multicolumn{2}{|c|}{2} & \multicolumn{2}{|c|}{3} & \multicolumn{2}{|c|}{4} & \multicolumn{2}{|c|}{5} & \multicolumn{2}{|c|}{6} & \multicolumn{2}{|c|}{7} & \multicolumn{2}{|c|}{8} & \multicolumn{2}{|c|}{9} & \multicolumn{2}{|c|}{10} & \multicolumn{2}{|c|}{11} & \multicolumn{2}{|c|}{12} \\
\hline & a & d & a & d & $a$ & d & a & d & $\mathbf{a}$ & d & $a$ & d & $a$ & D & $\mathbf{a}$ & d & a & d & $a$ & d & $a$ & $d$ & a & d \\
\hline Dia 1 & 3 & 3 & 3 & 3 & 3 & 2 & 2 & 2 & 2 & 2 & 3 & 3 & 2 & 2 & 2 & 2 & 2 & 2 & 2 & 2 & 2 & 2 & 2 & 2 \\
\hline Dia 2 & 3 & 3 & 3 & 4 & 3 & 2 & 3 & 3 & 2 & 2 & 2 & 2 & 2 & 2 & 2 & 2 & 2 & 2 & 2 & 2 & 2 & 2 & 1 & 1 \\
\hline Dia 3 & 3 & 3 & 3 & 3 & 2 & 2 & 2 & 2 & 2 & 2 & 2 & 3 & 2 & 2 & 2 & 2 & 2 & 2 & 2 & 2 & 2 & 2 & 1 & 1 \\
\hline
\end{tabular}

Tabela 3. Soma das respostas do questionário FIQ.

\begin{tabular}{lcc}
\hline \multicolumn{1}{c}{ Semana } & Soma de pontos & Melhora relativa $(\%)$ \\
\hline Pré & 60 & - \\
Quarta & 57 & $5 \%$ \\
Oitava & 38 & $36,7 \%$ \\
Décima-Segunda & 32 & $46,7 \%$ \\
\hline
\end{tabular}

No FIQ, dos 11 itens que avaliam a capacidade funcional em uma escala de 0 a 3 (nunca, ocasionalmente, na maioria das vezes, sempre). A voluntária melhorou em 8 itens (fazer compras, lavar roupas, lavar a louça, limpar a casa, arrumar a cama, caminhar várias quadras, visitar amigos ou parentes, dirigir automóvel e subir escadas) e relatou não sentir mais dificuldades na realização das tarefas diárias. Os demais itens não faziam parte da rotina diária da voluntária ou não apresentaram modificações. Com relação ao bem-estar, avaliado pelo número de dias em que a participante sentiu-se bem, houve melhora de 3 para 6 dias (melhora de $100 \%$ ) sem a presença exacerbada dos sintomas, ou um menor número de dias em que a voluntária deixou de trabalhar pela dificuldade de realizar tarefas devido a intensidade da dor (de 2 para nenhum dia de afastamento). Para os demais itens, destaca-se uma redução de 4 pontos na resposta de dor e uma melhora média de 2 graus nas respostas que implicou na redução de 46,7\% na pontuação total em relação à obtida inicialmente (de 60 para 32). 

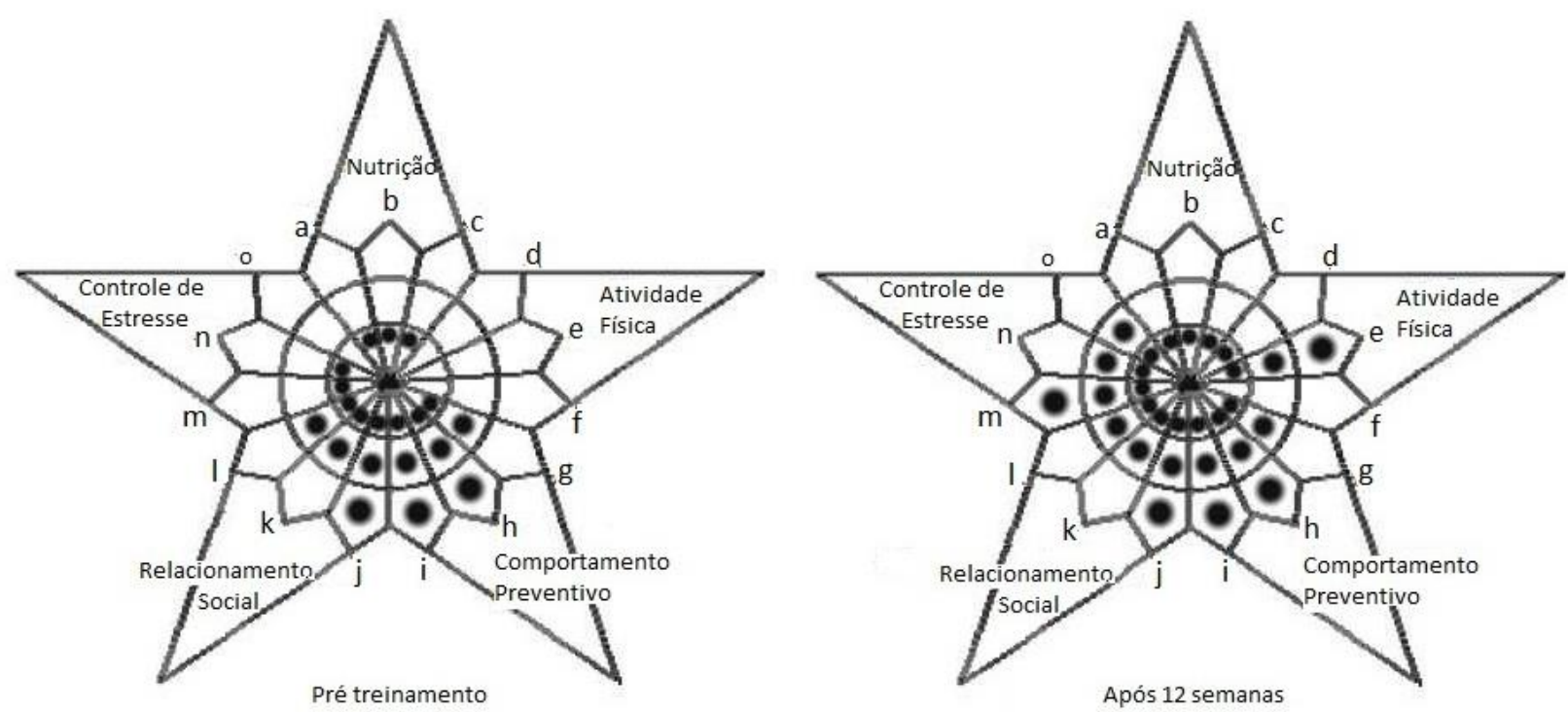

Figura 1. Respostas do questionário NAHAS.

Quanto ao questionário de NAHAS, dos 15 multifatores que determinam a qualidade de vida (classificados de nenhuma ocorrência a sempre), a voluntária apresentou melhoras em 5 deles (33\%). Destas respostas, duas se modificaram pelo próprio trabalho resistido ao qual a voluntária foi submetida $e$ as demais demonstraram alterações no controle dos fatores de estresse.

\section{Discussão}

O principal achado do presente trabalho foi mostrar que um período de 12 semanas de RML foi capaz de promover melhoria da qualidade de sono, da capacidade funcional, do bem-estar geral, do controle dos fatores de estresse e da qualidade de vida em uma voluntária do gênero feminino, com diagnóstico de FM por 2 anos, que não fazia uso de medicamentos e não realizava nenhum tipo de tratamento não medicamentoso específico contra FM. Deste modo, sabendo que a FM incorre em altos custos médicos diretos (BOONEN et al, 2005) e indiretos (licença por doença, afastamento remunerado por invalidez, etc) (LAMOTTE et al., 2010), torna-se importante buscar opções de tratamentos eficazes (HAUSER et al., 2010b).

O exercício físico tem se mostrado benéfico em pacientes com FM, pois além de melhorar a resistência cardiorrespiratória e muscular, já descritas exaustivamente na literatura, há evidencias recentes de que ele também promova alterações importantes nos circuitos neurais que modulam a dor (ELLINGSON et al., 2012).
Adicionalmente, observa-se na literatura que as mudanças neurogênicas, responsáveis pela sensibilização do sistema nervoso central, são tão importantes para modulação da dor quanto estímulos comportamentais, psicológicos ou ambientais (VAN WILGEN et. al., 2012).

Em um estudo recente, Ellingson et al. (2012) reportaram que a atividade física influencia o modo como o cérebro processa informações de dor sendo um possível mecanismo de alívio para pacientes com FM sugerindo que o estilo de vida sedentário pode piorar os efeitos deletérios da FM. Figueroa et al. (2008) reforçam este achado ao encontrarem associação entre disfunção autonômica pelo prejuízo do baroreflexo e a gravidade da FM.

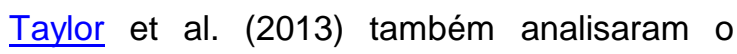
papel do sistema nervoso na dor e verificaram que a estimulação elétrica transcraniana foi capaz de reduzir a atividade neural nas regiões que modulam a dor sugerindo que a manipulação de circuitos centrais também pode estar associada com o alívio da dor. Esse argumento é reforçado pelos achados de Jensen et. al. (2012) que, através de estímulos de emoção e cognição por meio da terapia cognitivo comportamental, verificaram alteração da circuitaria neural responsável pela modulação da dor.

Além dos ajustes centrais na modulação da dor, observa-se também a importância dos fatores periféricos para tal modulação. Srikuea et al. (2013), por exemplo, observaram que as 
mudanças na musculatura esquelética advindas do sedentarismo associam-se com maior grau de fadiga em pacientes com FM.

Embora o presente não tenha tido objetivo de avaliar as influências neurais da dor, possivelmente as respostas favoráveis observadas na voluntária estão associadas à redução da dor e à melhora da sensibilidade periférica (baro, quimio e metabolo receptores) proporcionada pelo treinamento de físico (REYES DEL PASO et. al. 2011; FIGUEROA et. al. 2008).

Os benefícios na circuitaria neural decorrentes do treinamento de força podem proporcionar aos pacientes com FM uma nova condição muscular à qual determina um menor esforço para a execução das tarefas diárias gerando menos fadiga e menos dor, inclusive na situação de repouso (KINGSLEY et al., 2005). Tal eficiência muscular pode ser observada no presente trabalho através da análise da figura 1 e pelos relatos de redução do cansaço, da restrição na execução de trabalho e de dor na realização das tarefas rotineiras. Esta relação determina a melhora nos aspectos funcionais pelas modificações fisiológicas e resulta em maior disposição e independência nas atividades diárias (MAQUET et al., 2007).

Em conjunto, os estudos que investigaram adaptações neurais na FM sugerem que 0 exercício físico pode proporcionar benefícios não somente pelo estímulo mecânico que ele proporciona, mas também por estimular circuitos periféricos e centrais responsáveis pela modulação de dor.

Quanto ao questionário de $\mathrm{FIQ}$, no presente trabalho encontramos redução de $46 \%$ que assemelhou-se aos encontrados por Gowans et al., (1999) e Da Costa et al., (2005), contudo, outros estudos não demonstraram melhoras nos resultados do FIQ após prática de exercício em pacientes com FM (KING et al., 2002; KINGSLEY et al., 2005). Vale destacar que as divergências encontradas no escore de FIQ podem ser explicadas, pelo menos em parte, por diferenças metodológicas entre os estudos, pois alguns trabalhos foram feitos com cargas leves $e$ moderadas e outros com cargas mais intensas.

Quanto ao questionário de Nahas et al., (2000), a melhoria de controle do estresse também pode estar associada com redução da dor em função do treinamento resistido, que, segundo as modificações fisiológicas, pode facilitar a melhora psicossomática (OFFENBAECHER et al., 1999).

A literatura apresenta evidências sobre semelhanças entre os benefícios dos treinamentos de força e aeróbio (HOOTEN et al. 2012), porém, ainda necessita-se destas informações associadas à população com FM, para que haja quebra de preconceitos e 0 aumento nas possibilidades de práticas corporais efetivas para os portadores de FM. Apesar dos benefícios encontrados no presente trabalho, entendemos a limitação de relatar respostas de uma única avaliada. Apesar disso, acreditamos que nossos achados trazem importantes contribuições acerca do treinamento resistido para a melhora dos sintomas da FM e pode nortear não somente futuros estudos, mas também a prática profissional de sujeitos envolvidos com o tratamento não medicamentoso da FM.

Pelo exposto, apesar de nossos achados serem promissores, a realização de novos estudos que verifiquem os efeitos do treino resistido em portadores de FM e que sejam feitos de forma randomizada, com número amostral satisfatório, alto poder estatístico para diferentes desfechos, com grupo de intervenção e grupo controle e que permitam realizar comparações intra e entre grupos homogêneos ou heterogêneos quanto idade, gênero, nível de aptidão física e medicamentos utilizados será fundamental para maiores esclarecimentos.

\section{Conclusão}

O programa de 12 semanas de treinamento de RML aplicado a uma mulher de 52 anos, com 64 $\mathrm{Kg}$ de massa corpórea, $168 \mathrm{~cm}$ de estatura, IMC de 22,6, com diagnóstico de FM de 2 anos, foi capaz de reduzir o grau de influência da doença, mostrando-se favorável em aliviar os sinais e sintomas da FM. Na prática, tais resultados poderão ser de suma relevância para auxiliar tanto portadores de FM quanto profissionais que tratam e prescrevem treinos para este público.

\section{Referências}

ANG, D. C.; KALETH, A. S.; BIGATTI, S.; MAZZUCA, S.; SAHA, C.; HILLIGOSS, J.; LENGERICH, M.; BRANDY, R. Research to Encourage Exercise for Fibromyalgia (REEF): Use of motivational interviewing design and method.

Contemporary Clinical Trials, Indianápolis, v. 
32, n. 1 p. 59-68, 2011. doi:

http://dx.doi.org/10.1016/j.cct.2010.08.014

BELLATO, E.; MARINI, E.; CASTOLDI, F.; BARBASETTI, N.; MATTEI, L.; BONASIA, D.E.; BLONNA, D. Fibromyalgia syndrome: etiology, pathogenesis, diagnosis, and treatment. Pain Research and Treatment, Turin, v. 2012, ID. 426130, 2012. doi:

http://dx.doi.org/10.1155/2012/426130.

BOONEN, A.; VAN DEN HEUVEL, R.; VAN TUBERGEN, A.; GOOSSENS, M.; SEVERENS, J. L.; VAN DER HEIJDE, D.; VAN DER LINDEN, S. Large differences in cost of illness and wellbeing between patients with fibromyalgia, chronic low back pain, or ankylosing spondylitis. Annals of the Rheumatic Diseases, London, v. 64, n.3, p. 396-402, mar. 2005. doi: http://dx.doi.org/10.1136/ard.2003.019711

BURCKHARDT, C. S. Multidisciplinary approaches for management of fibromyalgia. Current Pharmaceutical Design, Schiphol, v.12, n. 1, p. 59-66, 2006. doi: http://dx.doi.org/10.2174/138161206775193217

BURCKHARDT, C. S.; GOLDENBERG, D.; CROFFORD, L.; GERWIN, R.; GOWANS, S.; JACKSON, K. et al. Guideline for the management of fibromyalgia syndrome: pain in adults and children. American Pain Society, Glenview (IL), 2005. doi: http://dx.doi.org/10.3238\%2Farztebl.2009.0383

BUSCH, A.; SCHACHTER, C.; OVEREDN, T.; PELOSO, P.; BARBER, K. Exercise for fibromyalgia: a systematic review. The Journal of Rheumatology, Toronto, v. 35, n.6, p. 1130-1144, 2008.

CARVILLE, S. F.; ARENDT-NIELSEN, S.; BLIDDAL, H.; BLOTMAN, F.; BRANCO, J. C.; BUSKILA, D.; DA SILVA, J.A.; DANNESKIOLDSAMSØE, B.; DINCER, F.; HENRIKSSON, C.; HENRIKSSON, K.G.; KOSEK, E.; LONGLEY, K.; MCCARTHY, G.M.; PERROT, S.;

PUSZCZEWICZ, M.; SARZI-PUTTINI, P.; SILMAN, A.; SPÄTH, M.; CHOY, E.H. EULAR evidence based recommendations for the management of fibromyalgia syndrome. Annals of the Rheumatic Disease, London, v. 67, n. 4, p. 536-41, 2008. doi: http://dx.doi.org/10.1136/ard.2007.071522

CROFFORD, L. J.; CLAUW, D. J. Fibromyalgia: where are we a decade after the American College of Rheumatology classification criteria were developed? Arthritis \& Rheumatism, Atlanta, v. 46, n. 5, p. 1136-1138, 2002. doi: http://dx.doi.org/10.1002/art.10217
DA COSTA, D.; ABRAHAMOWICZ, M.; LOWENSTEYN, I.; BERNATSKY, S.; DRITSA, M.; FITZCHARLES, M. A.; DOBKIN, P. L. A randomized clinical trial of an individualized homebased exercise programme for women with fibromyalgia. Rheumatology, Oxford, v. 44, n. 11, p. 1422-1427, 2005. doi: http://dx.doi.org/10.1093/rheumatology/kei032

EHRLICH, G. E. Pain is real; fibromyalgia isn't. The Journal of Rheumatology, Toronto, n.30, p. 1666-1667, 2003.

ELLINGSON, L.D.; SHIELDS, M.R.; STEGNER, A.J.; COOK, D.B. Physical activity, sustained sedentary behavior, and pain modulation in women with fibromyalgia. The Journal of Pain, Seattle, v.13, n. 2, p. 195-206, 2012. doi: http://dx.doi.org/10.1016/j.jpain.2011.11.001

FIGUEROA, A.; KINGSLEY, J. D.; MCMILLAN, V.; PANTON, L. B. Resistance exercise training improves heart rate variability in women with fibromyalgia. Clinical Physiology and

Functional Imaging, Oxford, v. 28, n.1, p. 49-54, 2008. doi: http://dx.doi.org/10.1111/i.1475097X.2007.00776.x

GILLILAND, B. C. Fibromyalgia, arthritis associated with systemic diseases and other arthritides. In: KASPER, D. L.; BRAUNWALD, E.; FAUCI, A.S. et al., editors. Harrison's principles of internal medicine. Nova York: McGraw-Hill, p. 2055-2056, 2005.

GOLDENBERG, D. L.; BURCKHARDT, C.; CROFFORD, L. Management of fibromyalgia syndrome. The Journal of American Medical Association, Barcelona, v. 292, n. 19, p. 23882395, 2004. doi:

http://dx.doi.org/10.1001/jama.292.19.2388.

GOWANS, S. E.; DEHUECK, A.; VOSS, S.; RICHARDSON, M. A randomized, controlled trial of exercise and education for individuals with fibromyalgia. Arthritis Care and Research, Atlanta, v.12, n. 2, p. 120-128, 1999.

GURSOY, S.; ERDAL, E.; HERKEN, H.; MADENCI, E.; ALAAEHIRLI, B.; ERDAL, N. Significance of catechol-Omethyltransferase gene polymorphism in fibromyalgia syndrome.

Rheumatology International, Berlin, v. 23, n. 3, p. 104-107, 2003. doi: http://dx.doi.org/10.1007/s00296-002-0260-5

HAKKINEN, A.; HAKKINEN, K.; HANNONEN, P.; ALEN, M. Strength training induced adaptations in neuromuscular function of premenopausal women with fibromyalgia: comparison with healthy women. Annals of the Rheumatic Disease, Oslo, Norway, v. 60, n. 1, p. 21-26, 2001. doi: http://dx.doi.org/10.1136\%2Fard.60.1.21 
HAUSER, W.; KLOSE, P.; LANGHORST, J.; MORADI, B.; STEINBACH, M.; SCHILTENWOLF, M.; BUSCH, A. Efficacy of different types of aerobic exercise in fibromyalgia syndrome: a systematic review and meta-analysis of randomised controlled trials. Arthritis Research and Therapy, London, v. 12, 2010a. doi: http://dx.doi.org/10.1186/ar3002

HAUSER, W.; THIEME, K.; TURK, D. C. Guidelines on the management of fibromyalgia syndrome - a systematic review. European Journal of Pain, London, v. 14, n. 1, p. 5-10, jan. 2010b. doi:

http://dx.doi.org/10.1016/j.ejpain.2009.01.006

HEYMANN, R. E.; PAIVA, E.; HELFENSTEIN JR, M.; POLLAK, D. F.; MARTINEZ, J. E.; PROVENZA, J. R. et al. Consenso brasileiro do tratamento de fibromialgia. Revista Brasileira de Reumatologia, Campinas, v. 50, n. 1, p. 56-66, 2010.

HOOTEN, W.M.; QU, W.; TOWNSEND, C.O.; JUDD, J.W. Effects of strength vs aerobic exercise on pain severity in adults with fibromyalgia: a randomized equivalent trial. Pain. Amsterdam, v. 153, n. 4, p. 915-923, 2012. doi: http://dx.doi.org/10.1016/i.pain.2012.01.020

HURLEY, B. F.; HANSON, E. D.; SHEAFF, A. K. Strength training as a countermeasure to aging muscle and chronic disease. Sports Medicine, Auckland, v. 41, n. 4, p. 289-306, abr. 2011. doi: http://dx.doi.org/10.2165/11585920-000000000$\underline{00000}$

JENSEN, K.B.; KOSEK, E.; WICKSELL, R.; KEMANI, M.; OLSSON, G.; MERLE, J.V.; KADETOFF, D.; INGVAR, M. Cognitive Behavioral Therapy increases pain-evoked activation of the prefrontal cortex in patients with fibromyalgia. Pain. Amsterdam, v. 153, n. 7, p.1495-503, 2012. doi: http://dx.doi.org/10.1016/j.pain.2012.04.010

JONES, K. D.; BURCKHARDT, C. S.; CLARK, S. R.; BENNETT, R. M.; POTEMPA, K. M. A randomized controlled trial of muscle strengthening versus flexibility training in fibromyalgia. The Journal of Rheumatology, Toronto, v. 29, n. 5, p. 1041-1048, 2002.

JONES, K. D.; LIPTAN, G. L. Exercise interventions in fibromyalgia: clinical applications from the evidence. Rheumatic Disease Clinics of North America, Pennsylvania, v. 35, n. 2, p. 373-391, mai. 2009. doi: http://dx.doi.org/10.1016/i.rdc.2009.05.004

KING, S. J.; WESSEL, J.; BHAMBHANI, Y.; SHOLTER, D.; MAKSYMOWYCH, W. The effects of exercise and education, individually or combined, in women with fibromyalgia. The
Journal of Rheumatology, Toronto, v. 29, n. 12, p. 2620-2627, dez. 2002.

KINGSLEY, J. D.; PANTON, L. B.; TOOLE, T.; SIRITHIENTHAD, P.; MATHIS, R.; MCMILLAN, V. The effects of a 12-week strength-training program on strength and functionality in women with fibromyalgia. Archives of Physical Medicine and Rehabilitation, Chicago, v. 86, n. 9, p. 17131721, set. 2005. doi: http://dx.doi.org/10.1016/j.apmr.2005.04.014

KOULIL, S. van; EFFTING, M.; KRAAIMAAT, F. W.; LANKVELD, W. van; HELMOND, T. van; CATS, H.; RIEL, P. L. C. M. van; JONG, A. J. L. de; HAVERMAN, J. F.; EVERS, A. W. M.

Cognitive-behavioural therapies and exercise programmes for patients with fibromyalgia: state of the art and future directions. Annals of the Rheumatic Disease, Oslo, v. 66, n. 5, p. 571581, 2007. doi:

http://dx.doi.org/10.1136/ard.2006.054692

LAMOTTE, M.; MAUGARS, Y.; LE LAY, K.; TAÏEB, C. Health economic evaluation of outpatient management of fibromyalgia patients and the costs avoided by diagnosing fibromyalgia in France. Clinical and Experimental

Rheumatology, Piza, v. 28, n. 6, supl. 63, dez. 2010.

LAWRENCE, R. C.; FELSON, D. T.; HELMICK, C. G.; ARNOLD, L. M.; CHOI, H.; DEYO, R. A.; GABRIEL, S.; HIRSCH, R.; HOCHBERG, M. C.; HUNDER, G. G.; JORDAN, J. M.; KATZ, J. N.; KREMERS, H. M.; WOLFE, F. Estimates of the Prevalence of Arthritis and Other Rheumatic Conditions in the United States Part II. Arthritis \& Rheumatism, Atlanta, v. 58, n. 1, p. 26-35, 2008. doi: http://dx.doi.org/10.1002/art.23176

MAQUET, D; DEMOULIN, C; CROISIER, J. L; CRIELAARD, J. M. Benefits of physical training in fibromyalgia and related syndromes. Annales de Réadaptation et de Médecine Physique, Amsterdam, v. 50, n. 6, p. 363-368, jul. 2007. doi: http://dx.doi.org/10.1016/j.annrmp.2007.03.021

MARQUES, A. P.; SANTOS, A. M. B.; ASSUMPÇAO, A.; MATSUTANI, L. A.; LAGE, L. V.; PEREIRA, C. A. B. Validação da Versão Brasileira do Fibromyalgia Impact Questionnaire (FIQ). Revista Brasileira de Reumatologia, Campinas, v. 46, n. 1, p. 24-31, jan/fev. 2006.

MARTINEZ-LAVÍN, M. Management of dysautonomia in fibromyalgia. Rheumatic Diseases Clinics of North America, v. 28, n. 2, p.379-387, mai. 2002.

MCNALLY, J. D.; MATHESON, D. A.; BAKOWSKY, V.S. The epidemiology of selfreported Fibromyalgia in Canada. Chronic 
Diseases in Canada, Ottawa, v. 27, n. 1, p. 9-16, 2006.

NAHAS, M. V.; BARROS, M. V. G.; FRANCALACCI, V. L. O Pentáculo do Bem-Estar. Base conceitual para avaliação do estilo de vida de indivíduos ou grupos. Revista Brasileira de Atividade Física e Saúde, Londrina, v. 5, n. 2, p. 48-59, 2000.

OFFENBAECHER, M.; BONDY, B.; DE JONGE, S.; GLATZEDER, K.; KRÜGER, M.; SCHOEPS, P.; ACKENHEIL, M. Possible association of fibromyalgia with a polymorphism in the serotonin transporter gene regulatory region. Arthritis \& Rheumatism, Atlanta, v. 42, n. 11, p. 2482-2488, 1999. doi: http://dx.doi.org/10.1002/15290131(199911)42:11<2482::AID-ANR27>3.0.CO;2B

PANTON, L. B.; KINGSLEY, J. D.; TOOLE, T.; CRESS, M. E.; ABBOUD, G.; SIRITHIENTHAD, P.; MATHIS, R.; MCMILLAN, V. A comparison of physical functional performance and strength in women with fibromyalgia, age and weight matched controls, and women who are healthy. Physical Therapy, New York, v. 86 , n. 11, p. 1479-1488, 2006. doi: http://dx.doi.org/10.2522/ptj.20050320

QIAO, Z. G.; VAEROY, H.; MORKRID, L. Electrodermal and microcirculatory activity in patients with fibromyalgia during baseline, acoustic stimulation and cold pressor tests. The Journal of Rheumatology, Toronto, v. 18, n. 9, p. 1383-1389, 1991.

RAJ, S. R.; BROUILLARD, D.; SIMPSON, C. S.; HOPMAN, W. M.; ABDOLLAH, H. Dysautonomia among patients with fibromyalgia: a noninvasive assessment. The Journal of Rheumatology, Toronto, v. 27, n. 11, p. 2660-2665, 2000.

REYES DEL PASO, G.A.; GARRIDO, S.; PULGAR, Á.; DUSCHEK, S. Autonomic cardiovascular control and responses to experimental pain stimulation in fibromyalgia syndrome. Journal of Psychosomatic Research, Oxford, v. 70, n.2, p. 125-134, 2011. doi:

http://dx.doi.org/10.1016/j.jpsychores.2010.09.012

RIBEIRO, K. L.; MARINHO, I. Fibromialgia e exercício. Fitness and Performance Journal, Rio de Janeiro, v. 4, n. 5, p. 280-287, 2005.

SANUDO, B.; GALIANO, D.; CARRASCO, L.; BLAGOJEVIC, M.; DE HOYO, M.; SAXTON, J. Aerobic Exercise Versus Combined Exercise Therapy in Women With Fibromyalgia Syndrome: A Randomized Controlled Trial. Archives of Physical Medicine and Rehabilitation, Chicago, v. 91 , n. 12 , p. $1838-1843$, dez. 2010. doi: http://dx.doi.org/10.1016/..apmr.2010.09.006
SRIKUEA, R.; SYMONS, T.B.; LONG, D.E.; LEE, J.D.; SHANG, Y.; CHOMENTOWSKI, P.J.; YU, G.; CROFFORD, L.J.; PETERSON, C.A.

Association of fibromyalgia with altered skeletal muscle characteristics which may contribute to postexertional fatigue in postmenopausal women. Arthritis and Rheumatism, Atlanta, v. 65, n 2, p. 519-528, 2013. doi: http://dx.doi.org/10.1002/art.37763

STAUD, R.; CANNON, R. C.; MAUDERLI, A. P.; ROBINSON, M. E.; PRICE, D. D.; VIERCK, C. J. JR. Temporal summation of pain from mechanical stimulation of muscle tissue in normal controls and subjects with fibromyalgia syndrome. Pain,

Amsterdam, v. 102, n. 1-2, p. 87-95, 2003. doi:

http://dx.doi.org/10.1016/s0304-3959(02)00344-5

TAYLOR, A.G.; ANDERSON, J.G.; RIEDEL, S.L.; LEWIS, J.E.; BOURGUIGNON, C. A randomized, controlled, double-blind pilot study of the effects of cranial electrical stimulation on activity in brain pain processing regions in individuals with fibromyalgia. Explore: The Journal of Science and Healing (NY). v. 9, n. 1, p. 32-40, 2013. doi: http://dx.doi.org/10.1016/..explore.2012.10.006

VALKEINEN, H.; HAKKINEN, K.; PAKARINEN, A.; HANNONEN, P.; HÄKKINEN, A.; AIRAKSINEN, O.; NIEMITUKIA, L.; KRAEMER, W. J.; ALÉN, M. Muscle hypertrophy, strength development, and serum hormones during strength training in elderly women with fibromyalgia. Scandinavian Journal of Rheumatology, Stockholm, v. 34, n. 4, p. 309314, ago. 2005. doi:

http://dx.doi.org/10.1080/03009740510018697

VAN WILGEN, C.P.; KEIZER, D. The sensitization model to explain how chronic pain exists without tissue damage. Pain Management Nursing. Philadelphia, v. 13 , n. 1, p. 60-65, 2012. doi: http://dx.doi.org/10.1016/i.pmn.2010.03.001

WIERWILLE, L. Fibromyalgia: diagnosing and managing a complex syndrome. Journal of the American Academy of Nurse Practitioners, Thorofarev. 24, n. 4, p. 184-192, 2012. doi: http://dx.doi.org/10.1111/j.17457599.2011.00671.x

WOLFE, F.; SMYTHE, H. A.; YUNUS, M. B.; BENNETT, R. M.; BOMBARDIER, C.; GOLDENBERG, D. L.; TUGWELL, P.; CAMPBELL, S. M.; ABELES, M.; CLARK, P. et al. The American College of Rheumatology 1990 criteria for the classification of fibromyalgia. Report of the Multicenter Criteria Committee. Arthritis and Rheumatism, Atlanta, v. 33, n. 2, p. 160-172, 1990. 


\section{Endereço:}

Cesar Cavinato Cal Abad

Estrada do Campo Limpo, 3.677 Campo Limpo.

São Paulo SP Brasil

05777-201

Telefone: (11) 58429000.

e-mail: c.cavinato@uol.com.br

Recebido em: 18 de março de 2012.

Aceito em: 9 de abril de 2013.

\footnotetext{
(c) (i)

Motriz. Revista de Educação Física. UNESP, Rio Claro,

SP, Brasil - elSSN: 1980-6574 - está licenciada sob Creative Commons - Atribuicão 3.0
} 